\title{
The geographies of perceived neighbourhood disorder: A small area estimation approach
}

DOI:

10.1016/j.apgeog.2019.102037

\section{Document Version}

Accepted author manuscript

Link to publication record in Manchester Research Explorer

\section{Citation for published version (APA):}

Buil-Gil, D., Medina, J., \& Shlomo, N. (2019). The geographies of perceived neighbourhood disorder: A small area estimation approach. Applied Geography, 109, [102037]. https://doi.org/10.1016/j.apgeog.2019.102037

\section{Published in:}

Applied Geography

\section{Citing this paper}

Please note that where the full-text provided on Manchester Research Explorer is the Author Accepted Manuscript or Proof version this may differ from the final Published version. If citing, it is advised that you check and use the publisher's definitive version.

\section{General rights}

Copyright and moral rights for the publications made accessible in the Research Explorer are retained by the authors and/or other copyright owners and it is a condition of accessing publications that users recognise and abide by the legal requirements associated with these rights.

\section{Takedown policy}

If you believe that this document breaches copyright please refer to the University of Manchester's Takedown Procedures [http://man.ac.uk/04Y6Bo] or contact uml.scholarlycommunications@manchester.ac.uk providing relevant details, so we can investigate your claim.

\section{OPEN ACCESS}




\title{
The geographies of perceived neighbourhood disorder. A small area estimation approach
}

\begin{abstract}
This research examines the geographical distribution of perceived neighbourhood disorder in Manchester, England, by using small area estimates. Sample surveys are the main source of information to analyse perceived disorder. However, most surveys are only representative of large areas, and direct estimates may be unreliable at small area level. Small area estimation techniques borrow strength from related areas to produce reliable small area estimates. This research produces Spatial Empirical Best Linear Unbiased Predictor (SEBLUP) estimates, which account for spatially correlated random area effects, of perceived neighbourhood disorder from the Manchester Resident Telephone Survey. The highest levels of perceived disorder are found in the city centre and some Northern and Central-Eastern areas. Perceived disorder is higher in areas with higher population churn, income deprivation and crime. Small area estimation techniques are a potential tool to map perceived disorder.
\end{abstract}

\section{Keywords}

Antisocial behaviour - Model-based estimation - Subjective security - Mapping Environmental criminology - EBLUP 


\section{Introduction}

Signs of disorder, observed and inferred, criminal and noncriminal, play a vital role in understanding wellbeing in contemporary cities. For residents, rowdy teenagers, panhandlers and public drinking, and even deteriorated housing and graffiti, can be perceived as disorderly and threatening. However, perceiving disorder goes beyond observed cues of lack of order and is shaped by neighbourhood stigmas and reputations as dimensions of social inequality (Sampson 2009; Taylor 2001). Factors such as the concentration of minorities and neighbourhood poverty are bound up by social meanings frequently associated with disorder (Franzini et al. 2008; Sampson and Raundenbush 1999; Wickes et al. 2013).

Perceived disorder erodes residential satisfaction and local commitment (Robinson et al. 2003) and is related to perceived powerlessness, fear and social mistrust (Ross and Mirowsky 1999; Ross et al. 2000; Skogan 2015). Moreover, Wilson and Kelling (1982) theorised that uncontrolled neighbourhood disorder causes an increase in serious crimes, leading to controversial zero tolerance policies (Skogan 1990; Taylor 2001).

Whether perceived disorder is seen as a cause of crime or as a socially damaging phenomenon itself, it is necessary to have an accurate picture of its geographical distribution. Maps of perceived disorder are useful to comprehend its causes and design evidenceinformed policies and policing interventions. As shown by Braga and Bond (2008), policing interventions that target hot spots of disorder and crime are successful in reducing crime and antisocial behaviour. However, while police data record crimes at a detailed geographical scale and allow for micro-level crime maps, more advanced statistical techniques are needed to map the neighbourhood disorder. Perceived disorder is mainly recorded by surveys, which are usually designed to be representative of large areas. Thus, direct estimates drawn from these are unreliable at small area level (Rao and Molina 2015). 
Furthermore, Hipp (2010a) shows that the ecological connections between socioeconomic variables (e.g. concentration of minorities, poverty), crime and neighbourhood perceptions operate at the scales of small communities or microneighbourhoods, rather than larger geographical units. Neighbourhood perceptions are shaped by immediate societal and environmental features and crimes and disorders happening within the neighbourhood (Hipp 2010a). Thus, we aim to reliably map the perceptions of disorder at small geographical level.

New methods have been explored to map disorder perceptions at low spatial levels, but these are limited by biases that could lead to unreliable maps. Crowdsourcing projects (Solymosi and Bowers 2018) and records of requests for city services (O'Brien et al. 2015) might be limited by little and biased social participation. Systematic social observation (SSO) may be limited by observer biases (Hoeben et al. 2016) and shows little consistency with perceptual measures of disorder (Yang et al. 2018).

In order to precisely map the perceived neighbourhood disorder, model-based small area estimation (SAE) techniques use existing survey data and introduce models to borrow strength across related areas (Rao and Molina 2015). In 2008, the US Panel to Review the Programs of the Bureau of Justice Statistics indicated the need for using SAE to produce regional estimates from the National Crime Victimization Survey (Groves and Cork 2008). This work was started by Fay and Diallo (2012) using area-level models with temporal random effects, which account for the temporal stability of crime trends but do not take advantage from the (typically) high spatial autocorrelation of crime and disorder. Others have explored different model-based synthetic estimators to map various attitudes towards crime (Taylor 2013; Wheeler et al. 2017); but synthetic estimators suffer from a risk of bias arising from the models (Rao and Molina 2015). 
In this work we suggest the application of the Spatial Empirical Best Linear Unbiased Predictor (SEBLUP) to estimate the residents' perceived disorder in their neighbourhoods. The SEBLUP accounts for the implicit spatial dimension of neighbourhood perceptions and is expected to provide better estimates than basic model-based estimators (Pratesi and Salvati 2008). This method has been applied to study poverty and social exclusion, but this is the first SEBLUP application to analyse neighbourhood perceptions. Mooney et al. (2018) suggest using a universal kriging, which also incorporates the spatial autocorrelation parameter and covariates in a model-based approach, to estimate physical disorder from Google Street View images. Although their approximation is innovative and incorporates the spatial dimension of disorder, it does not account for neither the neighbourhoods' stigmas experienced by residents in their areas nor the social disorder (observed or socially constructed), both of which have serious effects on residents' everyday life (Robinson et al. 2003; Ross and Mirowsky 1999).

This article introduces the SEBLUP to examine the geographies of a latent score of perceived disorder in Manchester Local Authority District (Manchester LAD), England. The SEBLUP is expected to overcome the main limitations found by previous research and produce reliable small area estimates, and it provides evidence about significant covariates to predict the distribution of perceived disorder. This is also one of the first applications that combine latent factor models and SAE. Section 2 presents the theoretical background and hypotheses. Section 3 introduces the data and estimation approaches, followed by results shown in section 4. Finally, section 5 presents discussion and conclusions. 


\section{Theoretical background}

\subsection{Perceived neighbourhood disorder}

Ross and Mirowsky (2001) define perceived neighbourhood disorder as "conditions and activities [...] that residents perceive to be signs of the breakdown of social order" (p. 265). Disorder is frequently classified into social and physical. Social disorder refers to episodic human behaviours that trouble citizens and indicate lack of social control; while physical disorder relates to time-persistent cues showing that an area is not maintained properly (Ross and Mirowsky 1999; Skogan 2015). Some cues indicating social disorder are street fights and public consumption of alcohol and drugs; and physical cues that might be perceived as disorderly are graffiti and rubbish lying around.

However, some authors argue that this distinction is unnecessary. Even when physical and social disorders are different problems that require specific policies, both drive to the same human reactions: "disorders in questions usually engender the same reaction -be it fight or flight- from neighborhood residents" (Skogan 1990, p. 4). Accordingly, Ward et al. (2017) examine perceived physical and social disorder from multilevel Structural Equation Modelling and argue that there is no utility in separating these when measuring perceived disorder at the neighbourhood level. This is the reason why we aim to produce estimates of a single latent score of perceived neighbourhood disorder.

\subsection{Neighbourhood characteristics and perceived disorder}

Even when neighbourhood perceptions are essentially individual, residents living in certain neighbourhoods perceive higher disorder than others. Perceived disorder has been associated with individual features such as age, ethnicity, victimisation and education level (Hipp 2010b; Steenbeek et al. 2012). However, perceptions of disorder are known to be especially influenced by community-level variables that shape social structures and neighbours' 
perceptions at a small geographical level, such as the concentration of minorities, poverty, unemployment, residential instability and crime (Hipp 2010b; Sampson and Raundenbush 2004; Steenbeek and Hipp 2011).

Sampson and Raudenbush $(1999,2004)$ analysed perceived disorder in Chicago from SSO and questionnaires and concluded that visual cues of lack of social and physical order partially predict perceived disorder, but the neighbourhood concentration of minorities, poverty and low social control have more explanatory power. Social cohesion and collective efficacy have shown a strong negative relation with both observed and perceived disorder (Sampson and Raudenbush 2001; Steenbeek and Hipp 2011; Taylor 2001). The neighbourhood's residential instability, as measured by the population churn of residents who move in and out, has been repeatedly associated to perceived disorder (Ross et al. 2000; Sampson and Raudenbush 1999; Steenbeek et al. 2012; Steenbeek and Hipp 2011). High crime rates are also known to increase the neighbours' perceived disorder (Franzini et al. 2008; McCord et al. 2007; Skogan 2015). With respect to the relation between minorities' concentration and perceived disorder, some argue that it is moderated by the neighbourhood's social cohesion (Wickes et al. 2013). Ross and Mirowsky (1999) found more perceived disorder in urban centres in Chicago, while citizens living in suburbs, villages and rural areas perceive less disorder; and Megler et al. (2014) found more graffiti reports in the central/commercial (mixed land-uses) areas of San Francisco. Mixed land-uses correspond to areas that enable different land uses, such as residential, commercial and leisure activity (e.g. central urban areas).

Perceived disorder is known to be especially affected by the characteristics of the micro-neighbourhood (Hipp 2010a). This paper introduces the SEBLUP to research on perceived disorder, to estimate its small geographies and analyse its predictors. The review of 
previous research provides essential information to decide which neighbourhood covariates should be fitted in SAE models.

\subsection{Hypotheses}

Based on the literature review, we expect to find higher perceived disorder in neighbourhoods characterised by:

H1. higher residential instability,

H2. lower income,

H3. higher crime rates,

H4. higher concentration of minorities,

H5. higher unemployment, and

H6. mixed land-uses.

Methodologically, we expect SEBLUP estimates to be more reliable than basic model-based estimates:

H7. SEBLUP estimates are more reliable than basic model-based estimates.

\section{Methods}

\subsection{Manchester Resident Telephone Survey (MRTS)}

This research is based on the MRTS, which recorded data between November 2012 and February 2014. The MRTS is a quota sample survey based on age, gender, ethnicity and employment, which is designed to measure general aspects of life in Manchester. After deleting households who refused to report their postcodes and respondents living outside Manchester LAD, our database has a sample of 7989 residents. Manchester is the major local authority of Greater Manchester Metropolitan County. The areas analysed in this research are the 282 Manchester Lower Layer Super Output Areas (LSOAs). LSOAs are small areas in 
England and Wales that contain between 1000 and 3000 residents, and between 400 and 1200 households. The average sample size per LSOA is $\bar{n}=28.3$, but samples vary between 2 and 79. Only 11 areas have less than 10 respondents, while most areas (143) have sample sizes between 10 and 29.

Prior research has shown that smaller units of analysis (e.g. street blocks) are preferred over larger scales when examining neighbourhood perceptions (Hipp 2010a). LSOAs are designed by grouping households which are physically and economically similar, show a good degree of overlap with urban communities, and are small enough to allow a close analysis of neighbourhood perceptions (Brunton-Smith et al. 2014). Furthermore, LSOAs are the smallest possible scales at which we are able to fit the area-level models and examine our hypotheses. Although MRTS data were recorded at a postcode level, some of the area-level covariates needed to fit the models are only available at LSOA level, and thus this research uses this unit of analysis.

\subsection{Variable of interest: Perceived disorder}

The MRTS includes questions about how much of a problem seven types of disorder are in one's local area (see Table 1). Respondents can answer 'Not a problem at all', 'Not a very big problem', 'A fairly big problem' and 'A very big problem'. 'No opinion' responses are recoded as missing. The measure with the lowest values is abandoned and burnt out cars, in which $93.3 \%$ of respondents reported that it was not a problem or not a very big problem; while rubbish lying around was reported as a fairly or very big problem by $44.2 \%$ of respondents. 
Table 1

Frequencies of measures of perceived disorder.

\begin{tabular}{lcccccc}
\hline & $\begin{array}{c}\text { Not a } \\
\text { problem at } \\
\text { all }\end{array}$ & $\begin{array}{c}\text { Not a very } \\
\text { big problem }\end{array}$ & $\begin{array}{c}\text { A fairly big } \\
\text { problem }\end{array}$ & $\begin{array}{c}\text { A very big } \\
\text { problem }\end{array}$ & $\begin{array}{c}\text { No } \\
\text { opinion }\end{array}$ \\
\hline Noisy neighbours and & $f$ & 5084 & 1651 & 840 & 383 & 31 \\
parties & $\%$ & $63.6 \%$ & $20.7 \%$ & $10.5 \%$ & $4.8 \%$ & $0.4 \%$ \\
Teenagers hanging & $f$ & 3939 & 1958 & 1315 & 730 & 47 \\
around the streets & $\%$ & $49.3 \%$ & $24.5 \%$ & $16.5 \%$ & $9.1 \%$ & $0.6 \%$ \\
Rubbish and litter lying & $f$ & 2606 & 1827 & 1965 & 1569 & 22 \\
around & $\%$ & $32.7 \%$ & $22.9 \%$ & $24.6 \%$ & $19.6 \%$ & $0.3 \%$ \\
Vandalism, graffiti and & $f$ & 4436 & 1900 & 1093 & 511 & 49 \\
damage to & $\%$ & $55.5 \%$ & $23.8 \%$ & $13.7 \%$ & $6.4 \%$ & $0.6 \%$ \\
property/vehicles & $f$ & 4118 & 1248 & 1232 & 804 & 587 \\
People using or dealing & $\%$ & $51.5 \%$ & $15.6 \%$ & $15.4 \%$ & $10.1 \%$ & $7.3 \%$ \\
drugs & $f$ & 4439 & 1937 & 1050 & 485 & 78 \\
People drunk or rowdy & $\%$ & $55.6 \%$ & $24.2 \%$ & $13.1 \%$ & $6.1 \%$ & $1 \%$ \\
in public places & $f$ & 6463 & 987 & 331 & 123 & 85 \\
Abandoned and burnt & $\%$ & $80.9 \%$ & $12.4 \%$ & $4.1 \%$ & $1.5 \%$ & $1.1 \%$ \\
out cars & & & & & & \\
\hline
\end{tabular}

In order to produce a unit-level single measure of perceived disorder, and given that all pairwise correlations between the seven measures were significant and strong, we hypothesised that all measures were underlying a single latent variable and computed a single latent score from Confirmatory Factor Analysis (CFA). The goodness-of-fit indicators suggest that the one-factor model is the best fitting CFA solution compared to the two-factor model (see Table 2). Measures of Root Mean Square Error of Approximation (RMSEA) and Root Mean Square of the Residuals (RMSR) are slightly smaller for the one-factor solution than the two-factor model (social and physical disorder), and the Tucker-Lewis Index (TLI) of factoring reliability is higher for the one-factor solution. The sum of squared factor loadings, which is the amount of variance explained by the single factor, is 2.72 for the one-factor solution. And the proportion of variance associated with each factor that could be explained from the dataset is 0.39 . Such measures are smaller for the two-factor solution. Moreover, the bivariate correlation between the two possible latent scores of social and physical disorder is very high $(\rho=0.97, p$-value $<0.01)$ and these load together on the same factor. 
Table 2

Goodness-of-fit indicators for one-factor and two-factor CFA solutions.

\begin{tabular}{lcc}
\hline & One-factor solution & Two-factors solution \\
\hline RMSEA & 0.05 & 0.07 \\
SMSR & 0.02 & 0.03 \\
TLI & 0.97 & 0.95 \\
Sum of squared factor loadings & 2.72 & 2.54 \\
Proportion of variance contributed by factors & 0.39 & 0.36 \\
\hline
\end{tabular}

Single latent scores were computed based on the Full Information Maximum Likelihood estimator, which makes maximal use of all data available from every respondent and can handle missing values, borrowing information about missing values on the basis of non-missing values, to obtain unbiased latent score estimates (Schlomer et al. 2010). All scores were shifted to a positive $0-1$ measure $\left(\frac{F_{i}-\min (F)}{\max (F)-\min (F)}\right)$ to make results easier to interpret (see Table 3), where $F_{i}$ is the latent score for unit $i$.

Table 3

Summary of latent scores and shifted latent scores of perceived disorder.

\begin{tabular}{lcccccc}
\hline & Minimum & $\begin{array}{c}\text { Lower } \\
\text { quartile }\end{array}$ & Median & Mean & $\begin{array}{c}\text { Upper } \\
\text { quartile }\end{array}$ & Maximum \\
\hline Latent scores & -0.99 & -0.77 & -0.19 & -0.01 & 0.53 & 3.21 \\
0-1 latent scores & 0 & 0.05 & 0.19 & 0.24 & 0.36 & 1 \\
\hline
\end{tabular}

Fig. 1 shows standardised loadings of disorder measures, which represent how these are weighted at the latent factor, and uniqueness values, which show the proportion of the variable common variance not associated with the factor. Some physical disorder indicators (rubbish and abandoned cars) have the lowest loadings; while measures of teenagers hanging around, people drunk and people using drugs, three social disorder indicators, have the highest loadings. Although the latent score of perceived disorder is composed of all measures of disorder and fits the data well, it gives more strength to indicators of social disorder than physical disorder measures. 


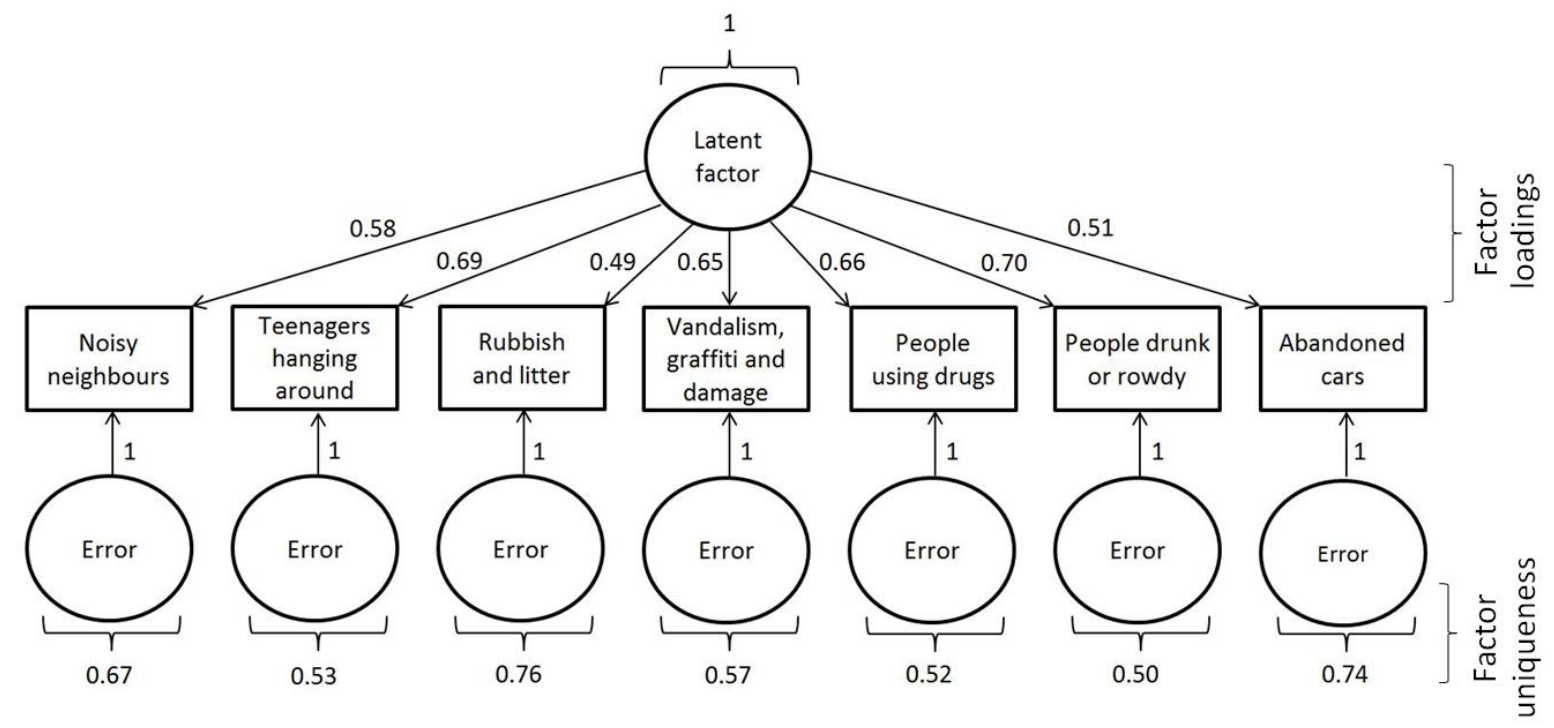

Fig. 1. Loadings and uniqueness for each indicator of the latent score of perceived disorder.

\subsection{Calculating survey weights}

Due to the use of quota sampling, we calibrate the sample to the population of Manchester to minimise bias arising from the non-random sampling design and to reduce possible biases arising from the decreased use of landlines. We calculate survey weights by calibrating the proportion of respondents according to certain characteristics to such proportion in the population. Let $N_{i}$ be the population count in class $i$ and similarly $n_{i}$ be the sample count. Then, the survey weight for respondents in class $i$ is $N_{i} / n_{i}$. In our case, the survey weights were calibrated by cross-classifying age (18-34, 35-54, 55+), sex (male, female), ethnicity (white, others) and area (North, Central, South Manchester). The weights calculation is limited by the social-demographic variables recorded by the survey. Variables such as respondents' country of birth were not included in the questionnaire and could not be included in the survey weights calibration. Population data were obtained from the UK Census 2011. Although the original sample characteristics were already quite similar to the population parameters due to the use of quota sampling, the use of survey weights adjusts the 
sample to the population characteristics and reduces the risk of bias in the final model-based estimates (see Table 4).

Table 4

Socio-demographic characteristics of MRTS sample and Manchester population (aged 18+).

\begin{tabular}{lccc}
\hline & $\begin{array}{c}\text { MRTS sample } \\
\text { (unweighted) }\end{array}$ & $\begin{array}{c}\text { MRTS sample } \\
\text { (weighted) }\end{array}$ & $\begin{array}{c}\text { Manchester LAD } \\
\text { population }\end{array}$ \\
\hline Age & & & \\
$18-34$ & $47.3 \%$ & $48.1 \%$ & $48.2 \%$ \\
$35-54$ & $30.6 \%$ & $30.1 \%$ & $30.1 \%$ \\
55 or more & $22.1 \%$ & $21.7 \%$ & $21.6 \%$ \\
Gender & & & \\
Male & $49.9 \%$ & $50.0 \%$ & $50.2 \%$ \\
Female & $50.1 \%$ & $50.0 \%$ & $49.8 \%$ \\
Ethnic origin & & & \\
White & $79.3 \%$ & $65.7 \%$ & $66.7 \%$ \\
Black & $4.6 \%$ & $7.9 \%$ & $8.5 \%$ \\
Asian & $12.4 \%$ & $19.5 \%$ & $17.1 \%$ \\
Other & $3.7 \%$ & $6.8 \%$ & $7.8 \%$ \\
Employment status & & & \\
Employed & $52.7 \%$ & $52.8 \%$ & $52.9 \%$ \\
Unemployed & $7.9 \%$ & $8.0 \%$ & $7.3 \%$ \\
Student & $12.2 \%$ & $13.1 \%$ & $13.3 \%$ \\
Retired & $12.7 \%$ & $12.1 \%$ & $12.3 \%$ \\
Other & $14.6 \%$ & $14.0 \%$ & $14.3 \%$ \\
\hline
\end{tabular}

\subsection{Auxiliary data}

In area-level model-based SAE, estimates borrow strength from a set of area-level covariates fitted in a model. Covariates were selected based on the literature review and preliminary data analyses. Then, we fitted an LSOA-level model with: (1) proportion of black and minority ethnic (BME) citizens 2011, (2) crime per capita 2012, (3) proportion of unemployed 2011, (4) income deprivation 2012, (5) population churn since 2011, and (6) a dichotomous 0-1 variable for mixed land-uses (see Table 5). 
Table 5

Summary of covariates and coefficients of correlation of each variable with direct estimates of perceived disorder.

\begin{tabular}{lccccccc}
\hline & Min & $\begin{array}{c}\text { First } \\
\text { quartile }\end{array}$ & Median & Mean & $\begin{array}{c}\text { Third } \\
\text { quartile }\end{array}$ & Max & $\begin{array}{c}\text { Spearman } \\
\text { coeff }\end{array}$ \\
\hline Proportion BME & 0.03 & 0.15 & 0.25 & 0.31 & 0.42 & 0.89 & $0.31^{* *}$ \\
Crime per capita & 0.04 & 0.11 & 0.16 & 0.17 & 0.23 & 6.04 & $0.29^{* *}$ \\
Proportion unemployed & 0.06 & 0.28 & 0.36 & 0.35 & 0.43 & 0.69 & $0.29^{* *}$ \\
Income deprivation & 0.01 & 0.12 & 0.24 & 0.24 & 0.34 & 0.52 & $0.35^{* *}$ \\
Population churn & 0.21 & 0.33 & 0.39 & 0.39 & 0.44 & 0.68 & $0.13^{*}$ \\
Mixed land-uses & 0 & 0 & 0 & 0.19 & 0 & 1 & $0.10^{*}$ \\
\hline
\end{tabular}

$* * p$-value $<0.01,{ }^{*} p$-value $<0.05$

The Income Deprivation score, which is recorded by the English Index of Multiple Deprivation 2015, measures the proportion of population with low salaries or without income. The proportion of BME and unemployed citizens were recorded by the UK Census 2011. Crimes per capita are computed from Greater Manchester Police data. Population churn is an estimate provided by the Consumer Data Research Centre which shows the proportion of households that changed its occupier between 2011 and 2016. The dichotomous variable of mixed land-uses is calculated based on the Classification of Multi-Dimensional Open Data of Urban Morphology, which describes the typology of each area based on its environmental and urban morphology attributes (Alexiou et al. 2016). Neighbourhoods defined as 'old town', 'high street and promenades' and 'central business district' are classified as mixed land-uses (coded as 1), while other categories are coded as 0 . This measure accounts only for morphological features and future research will explore the use of better measures of the level of mixed land-uses.

We also explored other variables, but their bivariate correlations with perceived disorder (as measured by direct estimates) were small or non-significant, so we did not include them in our models: mean age $(\rho=0.04, p$-value $>0.1)$, proportion of citizens not staying in school after $16(\rho=0.01, p$-value $>0.1)$, difference between the workday population and residents $(\rho=0.11, p$-value $>0.1)$, population density $(\rho=0.03, p$-value $>0.1)$ and premises licenced to sell alcohol $(\rho=0.07, p$-value $>0.1)$. Certain variables emphasised by literature, 
mainly social control and collective efficacy, could not be tested in our model, as we found no available data.

\subsection{Methodology}

We produce small area estimates of perceived disorder based on three SAE approaches: Horvitz-Thompson (HT) direct estimation, area-level EBLUP estimator, and EBLUP estimator with spatially correlated random effects (SEBLUP) (see Appendix for derivations).

First, the HT estimator uses data recorded by the original survey for each area and makes use of survey weights to produce design-unbiased direct estimates (Horvitz and Thompson 1952). Direct estimates might suffer from high variance and unreliability in areas with small sample sizes.

Second, the EBLUP estimator, which is based on the Fay-Herriot model (Fay and Herriot 1979), combines HT estimates with synthetic estimates produced from a linking model with area-level covariates to borrow strength from related areas (Rao and Molina 2015). Since the true values of the variable of interest are unknown, we use the HT estimates as our data, whose errors are different in each area because sample sizes vary between areas. We assume our direct estimates to be linearly related to a set of area-level covariates, fit a model and predict from it to compute the synthetic estimates. EBLUP estimates are produced from combining the HT estimates with synthetic estimates, with more weight attached to direct estimates when their error is small, and more weight given to synthetic estimates when direct estimates' error is large (Rao and Molina 2015). The EBLUP is an optimal combination between direct and synthetic estimates, and thus reduces the estimates' bias and is preferred over synthetic estimators. The fitting method chosen in this research is the restricted maximum likelihood, which takes into account the loss in degrees of freedom derived from the model (Rao and Molina 2015). 
Third, the SEBLUP estimator adds spatially correlated random area effects to EBLUP estimates and borrows strength from neighbouring areas through a simultaneous autoregressive process (Pratesi and Salvati 2008). The SEBLUP has shown to provide more reliable estimates than basic model-based estimators when the variable of interest shows medium-high levels of spatial autocorrelation, as is the case of neighbourhood perceptions (Hipp 2010a; Steenbeek et al. 2012). By borrowing strength from contiguous areas, we expect neighbouring areas to be more related than areas that do not share borders. The proximity matrix used in this research follows a standardised Queen Contiguity approximation, which defines as neighbouring areas not only polygons that share borders, but also areas that share at least one vertex. Queen Contiguity matrices are recommended in SAE when the number of areas under study is large (144 or more) (Asfar and Sadik 2016).

The main advantage of model-based SAE over other approaches to estimate population attributes at small area level, such as spatial microsimulation, is that extensive research has been devoted to the development of precise methods to examine the estimates' reliability (i.e. Relative Root Mean Squared Error, RRMSE). We note, however, that novel approaches are being explored to estimate uncertainty in spatial microsimulation approaches (Whitworth et al. 2017).

In SAE, estimates' RRMSEs are examined to analyse their level of reliability (a function of the variance and bias). Smaller RRMSEs indicate more reliable estimates. We expect a RRMSE reduction in EBLUP estimates in comparison to HT estimates. Furthermore, we expect RRMSEs to be reduced when producing SEBLUP estimates. RRMSEs are obtained by the method of bootstrapping adapted to account for variations arising from the CFA (Moretti et al. 2019) as follows:

For HT estimates, we draw $b=1, \ldots, 500$ simple random samples with replacement (SRSWR) from the sample based on the original sample sizes. In each sample we fit the CFA 
to predict factor scores. We then calculate survey weights and HT estimates in each sample. Finally, we calculate their average and bootstrap standard error. Dividing the bootstrap standard error by the average of direct estimates provides us with a bootstrap Coefficient of Variation, which is equivalent to the RRMSE for unbiased estimates.

The RRMSEs of EBLUP and SEBLUP estimates are produced using the parametric bootstrap procedure by Molina et al. (2009) adapted to account for the CFA (Moretti et al. 2019). We follow the steps described above: draw $b=1, \ldots, 500$ SRSWR, fit a CFA to predict factor scores, and calculate survey weights. Then, the parametric bootstrap is implemented on each sample according to steps described in Molina et al. (2009). We obtain a Monte Carlo unbiased approximation of the RRMSE of EBLUP and SEBLUP bootstrap estimates, which accounts for the variance arising from the CFA.

As a general rule, RRMSEs need to be lower than $25 \%$ to be regarded as reliable, RRMSEs ranging from $25 \%$ to $50 \%$ should be used with caution, and RRMSEs higher than $50 \%$ are unreliable (Rao and Molina 2015, p. 40). Estimates have been produced using the 'sae' package for $R$ software.

\section{Results}

In subsection 4.1 we discuss the model results. Subsection 4.2 presents the map of SEBLUP estimates. Subsection 4.3 checks the estimates' reliability and model diagnostics.

\subsection{The model}

Although the main objective of SAE is to produce estimates of increased reliability, the models fitted to produce such estimates provide relevant information about the covariates' explanatory capacity for understanding the distribution of the outcome measure. It is common practise in SAE applications to discuss their results. Table 6 shows the results of EBLUP and 
SEBLUP models used to produce the small area estimates of perceived disorder. AIC and BIC measures are slightly smaller in SEBLUP model than in EBLUP model, and SEBLUP estimates show the highest reliability measures (see subsection 4.3). Thus, SEBLUP model results are slightly preferred over EBLUP results.

Table 6

EBLUP and SEBLUP models of perceived disorder.

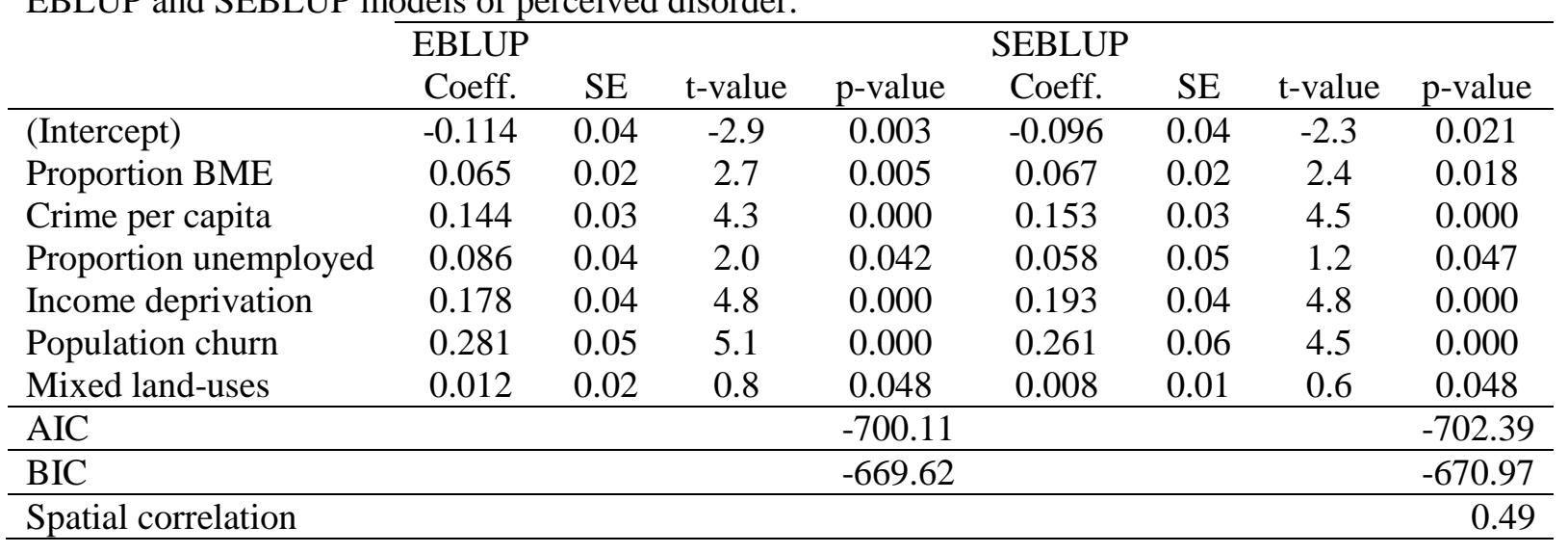

Both models show that population churn, the measure of residential instability, is the variable with the most predictive power of distribution of perceived disorder $\left(\beta^{E B L U P}=0.281\right.$ / $\beta^{S E B L U P}=0.261, p$-value $\left.<0.001\right)$. These coefficients provide strong evidence to accept our first hypothesis (H1). Income deprivation has the second strongest coefficient $\left(\beta^{E B L U P}=0.178\right.$ / $\beta^{\text {SEBLUP }}=0.193, p$-value $\left.<0.001\right)$. Thus, this variable needs to be considered when predicting perceived disorder $(\mathrm{H} 2)$. The number of crimes per capita also shows a significant positive relationship with perceived disorder $\left(\beta^{E B L U P}=0.144 / \beta^{S E B L U P}=0.153\right.$, $\mathrm{p}$-value $\left.<0.001\right)(\mathrm{H} 3)$. Finally, the concentration of BME (H4), unemployment (H5) and the measure of mixed landuses (H6) have significant but smaller positive relations with neighbourhood perceived disorder.

We observe very little variations between EBLUP and SEBLUP model coefficients. However, the measure of unemployment shows a clear smaller coefficient in the SEBLUP than in the EBLUP model. Non-spatial models can thus be seen to be overstating the 
contribution of unemployment to the explanation of the geographies of perceived disorder. Tvalues show lower values for the proportion of BME, population churn and mixed land-uses in SEBLUP than in EBLUP model. Non-spatial models might overestimate their effect to explain the distribution of perceived disorder. The spatial correlation coefficient is $\hat{\rho}=0.49$, which shows medium-high levels of spatial concentration.

\subsection{Mapping perceived disorder}

Direct, EBLUP and SEBLUP estimates have been produced (see Table 7). Since SEBLUP estimates are the most reliable estimates (see subsection 4.3), we analyse these and use them to produce the map in Fig. 2.

Table 7

Summary of small area estimates and average RRMSEs.

\begin{tabular}{lccccccc}
\hline & Minimum & $\begin{array}{c}\text { Lower } \\
\text { quartile }\end{array}$ & Median & Mean & $\begin{array}{c}\text { Upper } \\
\text { quartile }\end{array}$ & Maximum & $\begin{array}{c}\text { Average } \\
\text { RRMSE }\end{array}$ \\
\hline Direct & 0.001 & 0.124 & 0.175 & 0.184 & 0.235 & 0.629 & 28.50 \\
EBLUP & 0.001 & 0.131 & 0.166 & 0.166 & 0.204 & 0.437 & 20.41 \\
SEBLUP & 0.001 & 0.133 & 0.167 & 0.166 & 0.201 & 0.447 & 18.37 \\
\hline
\end{tabular}

Fig. 2 shows SEBLUP estimates at LSOA level in Manchester. Lighter shades of grey indicate lower estimates of perceived disorder, while darker areas correspond to higher perceptions. SEBLUP estimates show higher perceived disorder in most areas in Northern and Central-Eastern Manchester, while most Southern areas have lower estimates. The highest levels of perceived disorder have been estimated in the main commercial areas in the city centre (Megler et al. 2014; Ross and Mirowsky 1999). The highest estimate is located in the western area of the city centre $\left(\hat{\delta}^{S E B L U P}=0.45\right)$, where we find the central shopping mall (Arndale), Victoria train station, Manchester Arena concerts stadium and tourist attractions such as the John Rylands Library and National Football Museum. The second highest estimate is found in the southern area of the city centre $\left(\hat{\delta}^{S E B L U P}=0.35\right)$, where Piccadilly Gardens (main green area in city centre), Manchester Central coach station and the Gay 
Village are located. Manchester city centre is not only characterised by a very high population churn and crimes rate, but also by a large proportional difference between the usual residents and the workday population and a buoyant night-time economy. Large amounts of retail shops, business activity, historic buildings, green areas and main streets are characteristic of mixed land-uses. Most areas surrounding the central business and shopping hub in Manchester city centre have very low levels of perceived disorder, corresponding to neighbourhoods with lower crime and less poverty.

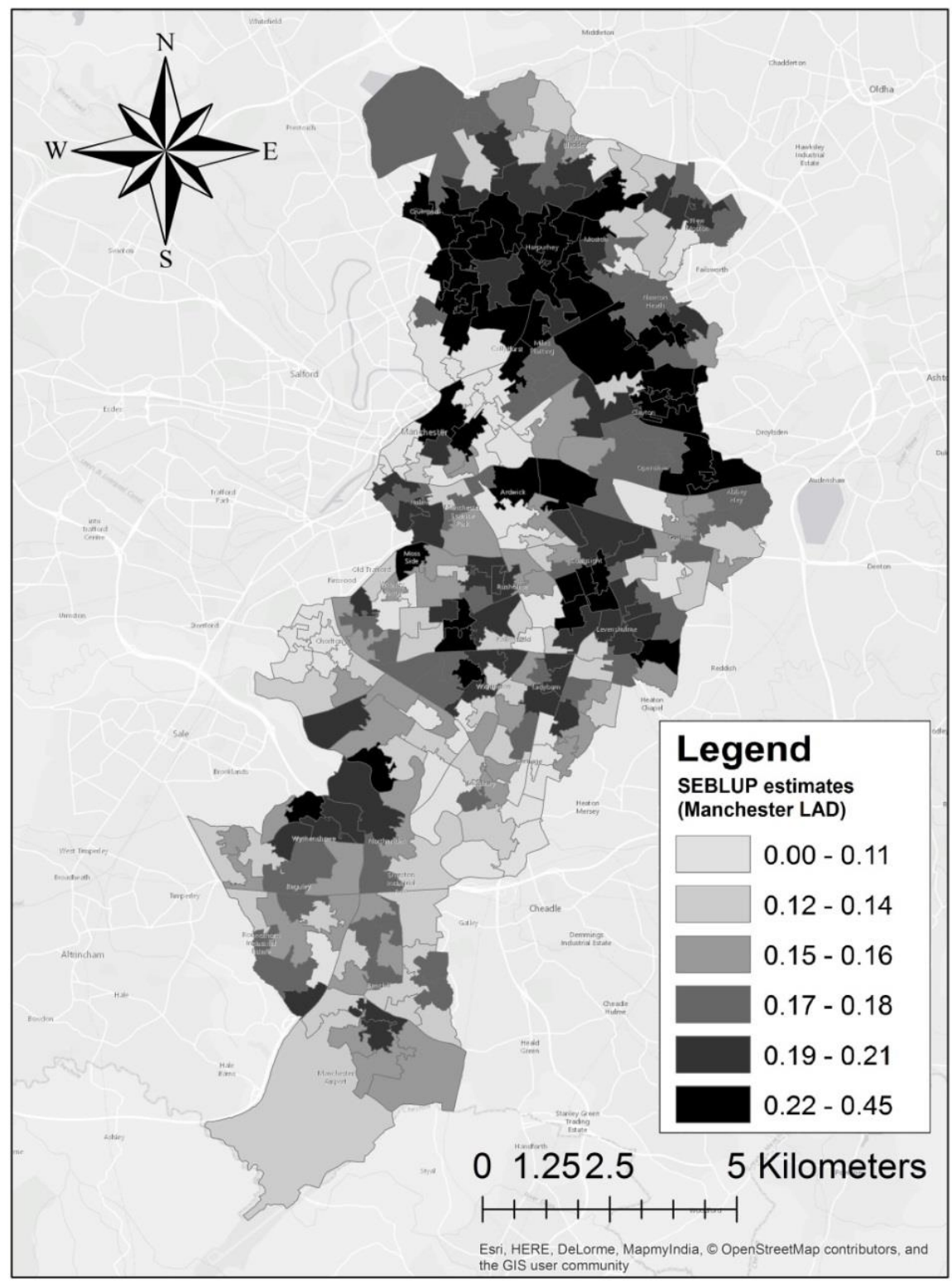

Fig. 2. SEBLUP estimates of perceived disorder in Manchester (division in 6 quantiles). 


\subsection{Checking the estimates' reliability and bias diagnostics}

RRMSE is the measure used in SAE to check the estimates' reliability, as a function of the variance and bias. SEBLUP estimates have the lowest average RRMSE (i.e. are the most reliable) (H7). Average RRMSE is reduced from $\overline{R R M S E}=28.50 \%$ of direct estimates to $\overline{R R M S E}=20.41 \%$ of EBLUPs and $\overline{R R M S E}=18.37 \%$ of SEBLUPs. Fig. 3 shows that RRMSEs have greatly decreased from direct to SEBLUP estimation in most areas: RRMSEs have only increased in two areas. Fig. 4 shows that most estimates were also improved from EBLUP to SEBLUP estimation: only 42 areas increased their RRMSEs, and none of such increases were greater than $3 \%$. While 172 direct estimates have RRMSEs higher than 25\%, only 39 areas have SEBLUP's RRMSEs greater than 25\%. Thus, RRMSEs of SEBLUP estimates are lower than $25 \%$ in most areas (243 out of 282), while a few number of LSOAs show values between 25 and $35 \%$ (31 out of 282), and only eight areas suffer from very low reliability (larger than 35\%). In general, these estimates show high reliability measures according to SAE standards (Rao and Molina 2015) and can be used for policy-making purposes. New methods for statistical testing are needed to examine whether differences between estimates in neighbouring areas (with their measures of error) are significant since the estimates are highly correlated due to the SAE spatial model. Such methods would evidence the utility of SAE for producing statistically distinguishable estimates but are currently out of scope of this paper. 


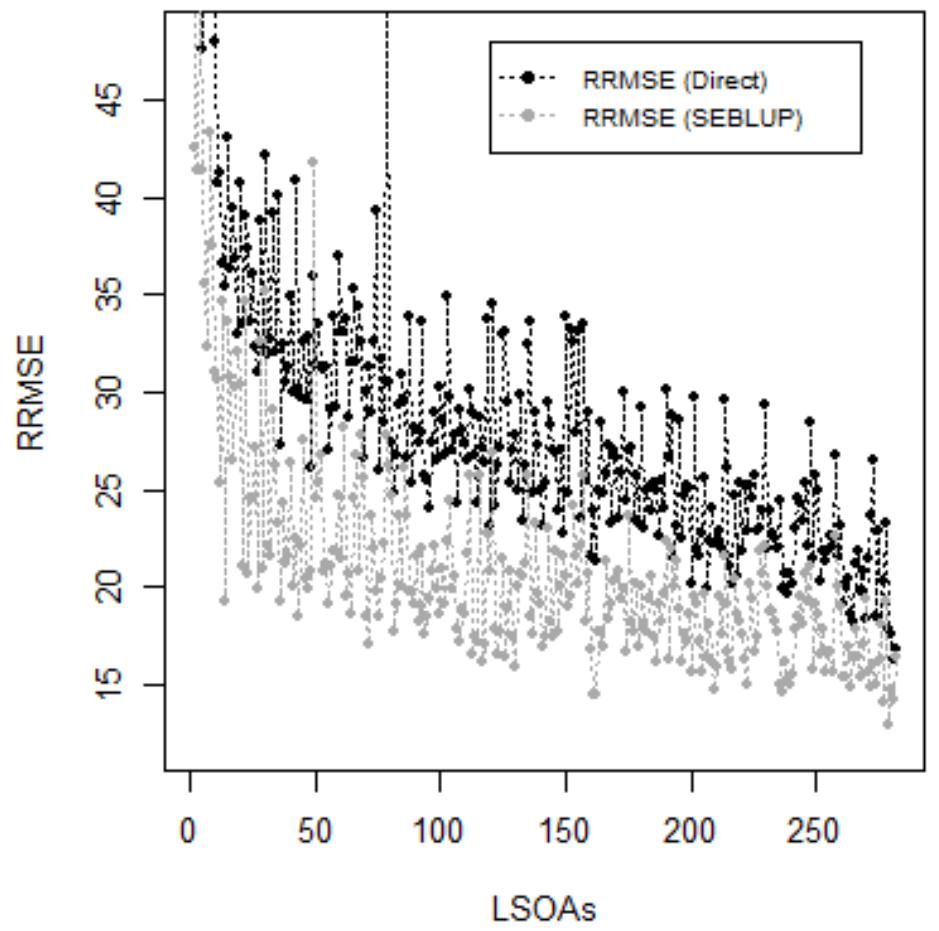

Fig. 3. RRMSEs of direct and SEBLUP estimates.

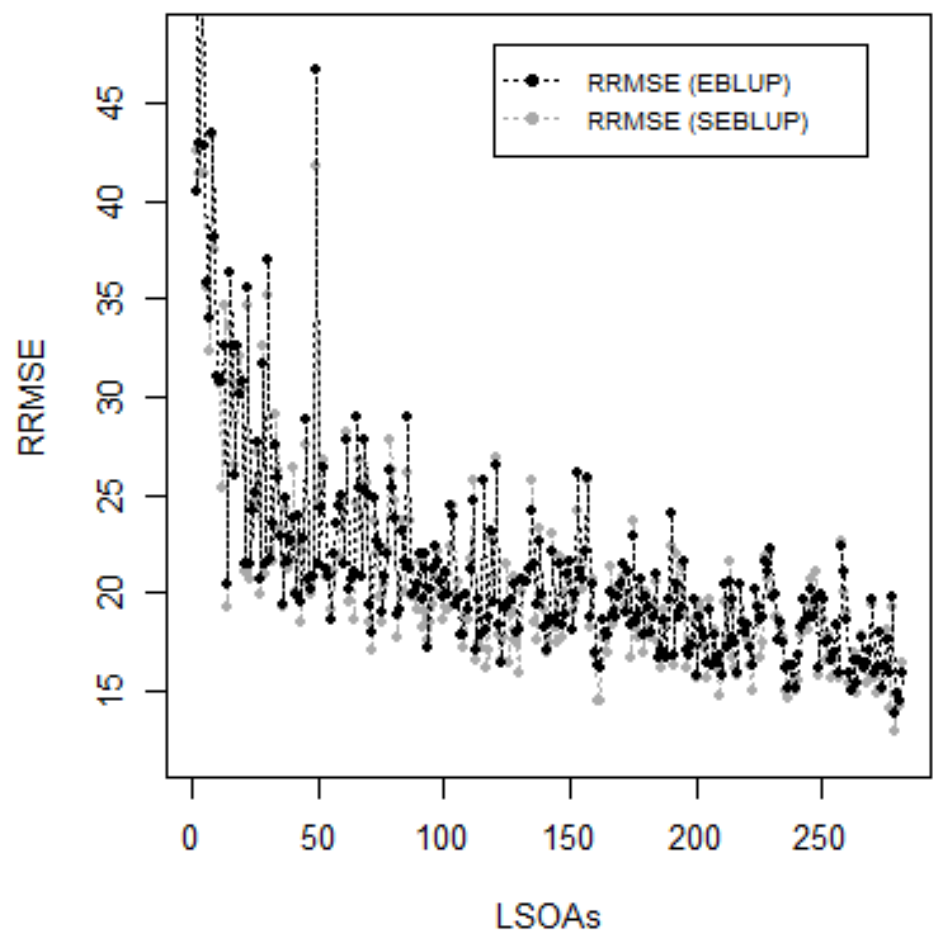

Fig. 4. RRMSEs of EBLUP and SEBLUP estimates. 
In relation to the model diagnostics, the Shapiro-Wilk test to check the normality of SEBLUP's standardised residuals suggests no rejection of the null hypothesis of normal distribution ( $\mathrm{W}=0.99, p$-value $=0.78$ ). The analytic validity of model-based estimates is examined by comparing these with the direct estimates, which are model-unbiased. We expect a high linear correlation between direct and SEBLUP estimates to show that modelbased estimates are not biased by the model. The Spearman coefficient of correlation is $\rho=0.87$ ( $p$-value $<0.001$ ), showing little bias coming from the model.

\section{Discussion and conclusions}

This research has introduced the SEBLUP to research on neighbourhood perceptions and has produced estimates of a single latent measure of perceived disorder for 282 LSOAs in Manchester. Goodness-of-fit indicators show that the single latent factor model is the best fitting solution and it allows for a single map of perceived disorder. Although geographical units smaller than LSOAs would be preferred to analyse neighbourhood perceptions (Hipp 2010a), LSOAs show a good degree of overlap with urban communities and are small enough to capture neighbourhood perceptions (Brunton-Smith et al. 2014), allowing to research our hypotheses. Our results illustrate that SEBLUP estimates are more reliable compared to other survey estimates, so we draw our discussion on their results. SEBLUP estimates have been used to examine the explanatory mechanisms and spatial distribution of citizens' perceived disorder in their own neighbourhoods, showing that residents' neighbourhood perceptions are related to the characteristics of their area of residence. App-based and crowdsourcing measures (Solymosi and Bowers 2018) might be appropriate for future research aiming to examine citizens' perceptions in areas other than their own neighbourhoods, but new methods are needed to overcome potential biases due to non-random sampling. 
The main methodological contribution of our research to previous research on perceived disorder is introducing the SEBLUP to analyse and map neighbourhood perceptions at a detailed geographical level. The SEBLUP takes advantage of the implicit spatial dimension of neighbourhood perceptions and produces more precise small area estimates than the EBLUP estimator (Pratesi and Salvati 2008). Such estimates allow for reliably mapping and targeting the hot spots of (perceived) disorder, leading to more accurate environmental explanations of neighbourhood perceptions. Reliable maps of perceived disorder are needed by crime analysts and police departments to develop strategic and intelligence-led tools, and to design and implement evidence-based micro-targeted policing practices and urban policies (Braga and Bond 2008).

The main substantive contribution of our research is adding compelling evidence about the relevance of residential instability, poverty and crime to explain the geographies of perceived disorder. Our models show that population churn and income deprivation are the two most important predictors of perceived neighbourhood disorder, and the correlation coefficient between these is very low $(\rho=0.03, p$-value $>0.5)$. Thus, the joint effect of poverty and residential instability largely explains the spatial distribution of perceived disorder in Manchester. Crime rates are also positively related to perceived neighbourhood disorder (Franzini et al. 2008; McCord et al. 2007); though some of the original measures of perceived disorder in the MRTS are regarded as crimes (e.g. drug dealing), and thus we expected some level of association (Skogan 2015).

Sampson and Raudenbush $(1999,2001)$ argue that residential instability might function as a structural condition that reduces social cohesion and collective efficacy, and in turn fosters disorder and crime. Furthermore, Ross et al. (2000) show that residential instability increases perceived disorder more in poor neighbourhoods with high crime rates than it does in wealthy areas: stability provides more advantages in affluent neighbourhoods. 
However, longitudinal data show that residential instability and disorder might be related in the opposite direction: disorder perceptions might encourage residents to move out, thus increasing residential instability (Sampson and Raudenbush 2001; Steenbeek and Hipp 2011). Further research and longitudinal data are needed to deepen the causal mechanisms between these, to examine whether perceived disorder is a direct consequence of poverty, residential instability and crime, as three independent constructs; or whether it functions as a mediator variable between the neighbourhood poverty, residential instability and crime.

Small but significant regression coefficients also indicate higher levels of perceived disorder in neighbourhoods with high concentration of minorities and unemployment and mixed land-uses (Ross and Mirowsky 1999; Sampson and Raundenbush 1999; Steenbeek et al. 2012). Mixed land-uses shows low model coefficients, partly due to the local nature of this study, and may show a larger effect in research analysing disorder at a supralocal level. Further research will seek better measures of the level of mixed land-uses, which might increase the models' explanatory power and estimates' reliability. Moreover, more research is needed to unmask the effect of the neighbourhood social cohesion on biased prejudices towards the presence of minorities, which may increase perceived disorder (Wickes et al. 2013). The use of alternative data sources should be explored to analyse the distribution of perceived disorder at lower spatial levels (e.g. output areas), which is expected to capture hidden internal heterogeneity in neighbourhood perceptions and result in less sharp boundaries -less dissimilar estimates- between neighbouring areas.

In conclusion, by introducing the SEBLUP to estimate the geographies of perceived disorder we are able to produce precise maps at a detailed spatial level and examine its main social organisation predictors. These findings allow local administrators and police departments to better understand neighbourhood perceptions and to design evidence-based 
micro-targeted interventions aimed to reduce crime and disorder and increase community safety.

\section{Acknowledgments}

The authors would like to thank the Manchester City Council for providing the survey data used in this research. Population churn data have been provided by the Consumer Data Research Centre, an ESRC Data Investment, under project ID CDRC 260, ES/L011840/1; ES/L011891/1. We thank Angelo Moretti and Reka Solymosi for comments that improved the manuscript. 


\section{Appendix. Definition of Horvitz-Thompson, EBLUP and SEBLUP estimators}

\section{Horvitz-Thompson estimator}

Let $U$ be the target population, which is divided into $D$ areas $U_{1}, \ldots, U_{D}$, of sizes $N_{1}, \ldots, N_{D}$. The Horvitz-Thompson direct estimator (Horvitz and Thompson 1952) for area $d$ is given by:

$$
\hat{\delta}_{d}^{D I R}=N_{d}^{-1} \sum_{i \in s_{d}} w_{d i} F_{d i}
$$

where $w_{d i}$ corresponds to the survey weight of unit $i$ from area $d$, and $F_{d i}$ is the latent factor score of perceived disorder of unit $i$ from area $d$.

\section{EBLUP}

The EBLUP based on the Fay-Herriot model (Fay and Herriot 1979) combines HT direct estimates and synthetic estimates produced from a linking model. We use the direct estimates $\hat{\delta}_{d}^{D I R}$, together with their errors $e_{d}$, and assume

$$
\hat{\delta}_{d}^{D I R}=\delta_{d}+e_{d}, \quad e_{d} \sim N\left(0, \psi_{d}\right), \quad d=1, \ldots, D,
$$

where $\psi_{d}$ refers to the sampling variance of direct estimates. We assume $\delta_{d}$ to be linearly related to a set of area-level covariates $\boldsymbol{x}_{\boldsymbol{d}}^{\prime}$,

$$
\delta_{d}=\boldsymbol{x}_{d}^{\prime} \boldsymbol{\beta}+v_{d}, \quad v_{d} \sim N(0, A), \quad d=1, \ldots, D,
$$

where $v_{d}$ is independent of $e_{d}$. Then, we obtain

$\hat{\delta}_{d}^{D I R}=\boldsymbol{x}_{d}^{\prime} \boldsymbol{\beta}+v_{d}+e_{d}, \quad v_{d} \sim N(0, A), \quad e_{d} \sim N\left(0, \psi_{d}\right), \quad d=1, \ldots, D$.

The Best Linear Unbiased Predictor (BLUP) of $\delta_{d}$ is given by

$$
\tilde{\delta}_{d}=\hat{\delta}_{d}^{D I R}-\frac{\psi_{d}}{A+\psi_{d}}\left\{\hat{\delta}_{d}^{D I R}-\boldsymbol{x}_{d}^{\prime} \widetilde{\boldsymbol{\beta}}(A)\right\}=\left\{1-\gamma_{d}(A)\right\} \hat{\delta}_{d}^{D I R}+\gamma_{d}(A) \boldsymbol{x}_{d}^{\prime} \widetilde{\boldsymbol{\beta}}(A),
$$


where $\gamma_{d}(A)=\psi_{d} /\left(A+\psi_{d}\right)$ and $\tilde{\beta}(A)$ is the maximum likelihood estimator of $\beta$. Since we do not know $A$, we replace it by an estimator $\hat{A}$ obtained from restricted maximum likelihood. When we modify $\hat{A}$ by $A$ we obtain the EBLUP (Rao and Molina 2015):

$$
\hat{\delta}_{d}^{E B L U P}=\left\{1-\gamma_{d}(\hat{A})\right\} \hat{\delta}_{d}^{D I R}+\gamma_{d}(\hat{A}) \boldsymbol{x}_{d}^{\prime} \widetilde{\boldsymbol{\beta}}(\hat{A}) .
$$

\section{SEBLUP}

The SEBLUP adds spatially correlated random area effects to EBLUP estimates (Pratesi and Salvati 2008). First, we consider the area-level model

$$
y=X \beta+v+e,
$$

where $\boldsymbol{y}$ is the vector of direct estimates $\left(\hat{\delta}_{1}^{D I R}, \ldots, \hat{\delta}_{D}^{D I R}\right)^{\prime}$ for D areas, $\boldsymbol{X}$ a matrix $\left(x_{1}, \ldots, x_{D}\right)^{\prime}$ of area-level explanatory variables for $\mathrm{D}$ areas, $\boldsymbol{v}$ a vector $\left(v_{1}, \ldots, v_{D}\right)^{\prime}$ of area effects and $\boldsymbol{e}$ a vector $\left(e_{1}, \ldots, e_{D}\right)^{\prime}$ of sampling errors independent of $\boldsymbol{v}$, with $\boldsymbol{e} \sim N\left(\mathbf{0}_{D}, \boldsymbol{\Psi}\right)$, where $\boldsymbol{\Psi}=\operatorname{diag}\left(\psi_{1}, \ldots \psi_{D}\right)$. We assume $\boldsymbol{v}$ to follow a SAR process with unknown autoregression parameter $\rho \in(-1,1)$ and a contiguity matrix $\boldsymbol{W}$ :

$$
\boldsymbol{v}=\rho \boldsymbol{W v}+\boldsymbol{u}
$$

We assume $\left(\boldsymbol{I}_{D}-\rho \boldsymbol{W}\right)$ to be non-singular, where $\boldsymbol{I}_{D}$ is referred to $D \times D$ identity matrix, so we can express

$$
\boldsymbol{v}=\left(\boldsymbol{I}_{D}-\rho \boldsymbol{W}\right)^{-1} \boldsymbol{u}
$$

where $\boldsymbol{u}=\left(u_{1}, \ldots, u_{D}\right)^{\prime}$ satisfies $\boldsymbol{u} \sim N\left(\mathbf{0}_{D}, A \boldsymbol{I}_{D}\right)$ for an unknown $A$. Then, we obtain

$$
y=X \beta+\left(I_{D}-\rho W\right)^{-1} u+e .
$$

The vector of variance components is now denoted as $\boldsymbol{\theta}=\left(\theta_{1}, \theta_{2}\right)^{\prime}=(A, \rho)^{\prime}$. The spatial BLUP of $\delta_{d}=\boldsymbol{x}_{\boldsymbol{d}}^{\prime} \boldsymbol{\beta}+v_{d}$ is given by

$$
\tilde{\delta}_{d}^{S B L U P}(\boldsymbol{\theta})=\boldsymbol{x}_{\boldsymbol{d}}^{\prime} \widetilde{\boldsymbol{\beta}}(\boldsymbol{\theta})+\boldsymbol{b}_{\boldsymbol{d}}^{\prime} \boldsymbol{G}(\boldsymbol{\theta}) \boldsymbol{\Sigma}^{-\mathbf{1}}(\boldsymbol{\theta})\{\boldsymbol{y}-\boldsymbol{X} \widetilde{\boldsymbol{\beta}}(\boldsymbol{\theta})\},
$$


where $\boldsymbol{b}_{\boldsymbol{d}}^{\prime}$ is a $1 \times d$ vector $(0, \ldots, 1,0, \ldots, 0)$ with 1 in position $d, \boldsymbol{G}(\boldsymbol{\theta})$ is the covariance matrix of $\boldsymbol{v}$ defined as $\boldsymbol{G}(\boldsymbol{\theta})=A\left\{\left(\boldsymbol{I}_{\boldsymbol{D}}-\boldsymbol{\rho} \boldsymbol{W}\right)^{\prime}\left(\boldsymbol{I}_{\boldsymbol{D}}-\boldsymbol{\rho} \boldsymbol{W}\right)\right\}^{-\mathbf{1}}, \boldsymbol{\Sigma}(\boldsymbol{\theta})$ is the covariance matrix of $\boldsymbol{y}$ obtained as $\boldsymbol{\Sigma}(\boldsymbol{\theta})=\boldsymbol{G}(\boldsymbol{\theta})+\boldsymbol{\Psi}$, and $\widetilde{\boldsymbol{\beta}}(\boldsymbol{\theta})$ is the weighted least squares estimator of $\boldsymbol{\beta}$ defined as $\widetilde{\boldsymbol{\beta}}(\boldsymbol{\theta})=\left\{\boldsymbol{X}^{\prime} \boldsymbol{\Sigma}^{-\mathbf{1}}(\boldsymbol{\theta}) \boldsymbol{X}\right\}^{-\mathbf{1}} \boldsymbol{X}^{\prime} \boldsymbol{\Sigma}^{-\mathbf{1}}(\boldsymbol{\theta}) \boldsymbol{y}$ (Pratesi and Salvati 2008). If we replace a consistent estimator of $\widehat{\boldsymbol{\theta}}=(\hat{A}, \hat{\rho})^{\prime}$ by $\boldsymbol{\theta}$ we obtain the SEBLUP:

$$
\hat{\delta}_{d}^{S E B L U P}=\tilde{\delta}_{d}^{S E B L U P}(\widehat{\boldsymbol{\theta}})=\boldsymbol{x}_{\boldsymbol{d}}^{\prime} \widetilde{\boldsymbol{\beta}}(\widehat{\boldsymbol{\theta}})+\boldsymbol{b}_{\boldsymbol{d}}^{\prime} \boldsymbol{G}(\widehat{\boldsymbol{\theta}}) \boldsymbol{\Sigma}^{-\mathbf{1}}(\widehat{\boldsymbol{\theta}})\{\boldsymbol{y}-\boldsymbol{X} \widetilde{\boldsymbol{\beta}}(\widehat{\boldsymbol{\theta}})\} .
$$




\section{References}

Alexiou, A., Singleton, A., \& Longley, P.A. (2016). A classification of multidimensional open data of urban morphology. Built Environment 42(3):382-295.

Asfar, A.K., \& Sadik, K. (2016). Optimum spatial weighted in small area estimation. Global Journal of Pure and Applied Mathematics 12(5):3977-1989.

Braga, A.Y., \& Bond, D.J. (2008). Policing crime and disorder hot spots: A randomized controlled trial. Criminology 46(3):577-607.

Brunton-Smith, I., Jackson, J., \& Sutherland, A. (2014). Bridging structure and perception: On the neighbourhood ecology of beliefs and worries about violent crime. British Journal of Criminology 54(4):503-526.

Fay, R.E., \& Herriot, R.A. (1979). Estimates of income for small places. An application of JamesStein procedures to census data. Journal of the American Statistical Association 74:269-277.

Fay, R.E., \& Diallo, M.S. (2012). Small area estimation alternatives for the National Crime Victimization Survey. In American Statistical Association (Ed.) Proceedings of the Survey Research Methods Section (pp. 3742-3756).

Franzini, L., O’Brien Caughy, M., Murray Nettles, S., \& O'Campo, P. (2008). Perceptions of disorder: Contributions of neighborhood characteristics to subjective perceptions of disorder. Journal of Environmental Psychology 28:83-93.

Groves, R.M., \& Cork, D.L. (Eds.) (2008). Surveying victims: Options for conducting the National Crime Victimization Survey. Washington: National Academies Press.

Hipp, J.R. (2010a). What is 'neighbourhood' in neighbourhood satisfaction? Comparing the effects of structural characteristics measured at the micro-neighbourhood and tract levels. Urban Studies 47(12):2517-2536.

Hipp, J.R. (2010b). Resident perceptions of crime and disorder: How much is "bias", and how much is social environment differences? Criminology 48(2):475-508. 
Hoeben, E., Steenbeek, W., \& Pauwels, L.J.R. (2016). Measuring disorder: observer bias in systematic social observation at streets and neighborhoods. Journal of Quantitative Criminology 34(1):221-249.

Horvitz, D.G., \& Thompson, D.J. (1952). A generalization of sampling without replacement from a finite universe. Journal of the American Statistical Association 47(260):663-685.

McCord, E.S., Ratcliffe, J.H., Garcia, R.M., \& Taylor, R.B. (2007). Nonresidential attractors and generators elevate perceived neighborhood crime and incivilities. Journal of Research in Crime and Delinquency 44(4):295-320.

Megler, V., Banis, D., \& Chang, H. (2014). Spatial analysis of graffiti in San Francisco. Applied Geography 54:63-73.

Molina, I., Salvati, N., \& Pratesi, M. (2009). Bootstrap for estimating the MSE of the Spatial EBLUP. Computational Statistics 24:441-458.

Mooney, S.J., Bader, M.D.M., Lovasi, G.S., Neckerman, K.M., Rundle, A.G., \& Teitler, J.O. (2018). Using universal kriging to improve physical disorder measurement. Sociological Methods \& Research. https://doi.org/10.1177/0049124118769103

Moretti, A., Shlomo, N., \& Sakshaug, J. (2019). Small area estimation of latent economic well-being. Sociological Methods \& Research. https://doi.org/10.1177/0049124119826160

O’Brien, D.T., Sampson, R.J., \& Winship, C. (2015). Ecometrics in the age of big data: Measuring and assessing "broken windows" using large-scale administrative data. Sociological Methodology 45(1):101-147.

Pratesi, M., \& Salvati, N. (2008). Small Area Estimation: The EBLUP estimator based on spatially correlated random area effects. Statistical Methods \& Applications 17:113-141.

Rao, J.N.K., \& Molina, I. (2015). Small area estimation. Second edition. Hoboken: Wiley.

Robinson, J.B., Lawton, B.A., Taylor, R.B., \& Perkins, D.D. (2003). Multilevel longitudinal impacts of incivilities: Fear of crime, expected safety, and block satisfaction. Journal of Quantitative Criminology 19(3):237-274.

Ross, C.E., \& Mirowsky, J. (1999). Disorder and decay: The concept and measurement of perceived neighborhood disorder. Urban Affairs Review34: 412-432. 
Ross, C.E., \& Mirowsky, J. (2001). Neighborhood disadvantage, disorder, and health. Journal of Health and Social Behavior 42(3):258-276.

Ross, C.E., Reynolds, J.R., \& Geis, K.J. (2000). The contingent meaning of neighborhood stability for residents’ psychological well-being. American Sociological Review 65(4):581-597.

Sampson, R.J. (2009). Disparity and diversity in the contemporary city: social (dis)order revisited. British Journal of Sociology 60(1):1-31.

Sampson, R.J., \& Raudenbush, S.W. (1999). Systematic social observation of public spaces: A new look at disorder in urban neighborhoods. American Journal of Sociology 105(3):603-651.

Sampson, R.J., \& Raudenbush, S.W. (2001). Disorder in urban neighborhood - Does it lead to crime? Washington DC: National Institute of Justice.

Sampson, R.J., \& Raudenbush, S.W. (2004). Seeing disorder: Neighborhood stigma and the social construction of "broken windows". Social Psychology Quarterly 67(4):319-342.

Schlomer, G., Bauman, S., \& Card, N. (2010). Best practices for missing data management in counseling psyschology. Journal of Counselling Psychology 57(1):1-10.

Skogan, W.G. (1990). Disorder and decline: Crime and the spiral of decay in American neighborhoods. Berkeley: University of California Press.

Skogan, W.G. (2015). Disorder and decline: The state of research. Journal of Research in Crime and Delinquency 52(4):464-485.

Solymosi, R., \& Bowers, K. (2018). The role of innovative data collection methods in advancing criminological understanding. In G.J.N. Bruinsma and S.D. Johnson (Eds.) The Oxford handbook of environmental criminology (pp. 210-237). Oxford: Oxford University Press.

Steenbeek, W., \& Hipp, J.R. (2011). A longitudinal test of social disorganization theory: Feedback effects among cohesion, social control, and disorder. Criminology 49(3):833-871.

Steenbeek, W., Völker, B., Flap, H., \& Oort, F. (2012). Local business as attractors or preventers of neighborhood disorder. Journal of Research in Crime and Delinquency 49(2):213-248.

Taylor, J. (2013). Small area synthetic estimation of perceptions of alcohol and drug-related antisocial behaviour. $\mathrm{PhD}$ thesis, University of Portsmouth, Portsmouth, UK.

Taylor, R.B. (2001). Breaking away from broken windows. Boulder: Westview Press. 
Ward, J.W., Link N.W., \& Taylor, R.B. (2017). New windows into a broken construct: A multilevel factor analysis and DIF assessment of perceived incivilities. Journal of Criminal Justice $51: 74-88$.

Wheeler, A., Silver, J., Worden, R., \& Mclean, S. (2017). Mapping attitudes towards the police at micro places. Available online at: http://dx.doi.org/10.2139/ssrn.3079674

Whitworth, A., Carter, E., Ballas, D., \& Moon, G. (2017). Estimating uncertainty in spatial microsimulation approaches to small area estimation: A new approach to solving an old problem. Computers, Environment and Urban Systems 63:50-57.

Wickes, R., Hipp, J.R., Zahnow, R., \& Mazerolle, L. (2013). “Seeing” minorities and perceptions of disorder: Explicating the mediating and moderating mechanisms of social cohesion. Criminology 51(3):519-560.

Wilson, J.Q., \& Kelling, G.L. (1982). Broken windows: The police and neighborhood safety. The Atlantic Magazine, March 1982.

Yang, S.M., Hinkle, J.C., \& Wyckoff, L.A. (2018). Using Multitrait-Multimethod (MTMM) techniques to examine the convergent and discriminant validity of social disorder. Journal of Research in Crime and Delinquency 55(5):571-608. 